Enf Neurol (Mex)

Vol. 12, No. 1: 45-47, 2013

Ensayo

CINNN, 2010

\title{
Breves aspectos de la enfermería según Florence Nightingale
}

\author{
María Elena Rodríguez Raso,* Sandra Hernández Corral**
}

\begin{abstract}
RESUMEN
Florence Nightingale, antecesora de la enfermería, publicó una de sus obras más importantes, Notas sobre enfermería: Qué es, qué no es. Las ideas plasmadas en su texto permiten reconocer los fundamentos de la enfermería moderna. En este trabajo se trata de reinterpretar algunas de sus notas, tales como el cuidado, la enfermería, su vocación, la observación, la administración y la influencia del entorno saludable sobre los individuos.
\end{abstract}

Palabras clave: Enfermería, cuidado, entorno, salud.

\section{Brief aspects of nursing as Florence Nightingale}

\begin{abstract}
Florence Nightingale, predecessor of nursing, published one of his most important works, Notes on Nursing: What it is, what it is not. The ideas in the text can recognize the fundamentals of modern nursing. This paper seeks to reinterpret some of his notes such as care, nursing, vocation, observation, administration and environmental influences on healthy individuals.
\end{abstract}

Key words: Nursing, care, environment, health.

$\mathrm{F}$ Tlorence Nightingale (1820-1910), precursora de la enfermería moderna, se destacó desde muy joven en matemáticas, y aplicó sus conocimientos de estadística a la epidemiología y a la estadística sanitaria. ${ }^{1}$ Concentró su inteligencia y su voluntad para romper con las tradiciones victorianas. Utilizó su perseverancia y persistencia al vivir una vida moderna sirviendo a la humanidad.

En 1859 publicó su libro Notas sobre Enfermería: Qué es y qué no es. Éste ha cumplido 154 años desde su primera edición. Las palabras "qué es y qué no es" son profundas y sustanciales, en donde se puede percibir qué es lo específico de la enfermera y cuáles son sus límites, además de la responsabilidad personal de ésta en el bienestar del paciente; desde esos tiempos, la autora anunciaba un saber y hacer propios de la disciplina.

Florence Nightingale menciona en su libro: "De ninguna manera estas notas tienen la intención de ser una regla de pensamiento por la cual las enfermeras pueden enseñarse a sí mismas a cuidar, ni mucho menos un manual para enseñar a las enfermeras el arte de cuidar. Están dirigidas sencillamente a dar algunas ideas a mujeres que tienen el cargo personal de la salud de otros."

Desde esos tiempos, perfila a las enfermeras con criterios, conocimientos y decisiones propias; recordemos que el cuidar surge como un acto de vida propio de la existencia del hombre, por lo cual, el cuidar estaba confinado a las

* Enfermera Jefe de Servicio del Instituto Nacional de Rehabilitación. Enfermera especialista del Hospital 20 de Noviembre, ISSSTE. ** Subjefe de Educación e Investigación en Enfermería del Instituto Nacional de Rehabilitación.

Correspondencia: María Elena Rodríguez, Raso, Calzada México-Xochimilco Núm. 289, Colonia Arenal de Guadalupe, 14389, Delegación Tlalpan. Teléfono: 59991000 ext. 12230. E-mail: rodriguez_elena72@yahoo.com.mx 
mujeres; pero el cuidado se ha transformado con la historia. El cuidar ahora es una actividad profesional; la enfermería también ha evolucionado según el contexto histórico. La autora menciona que la enfermería era mucho más de lo que ella vislumbraba en ese momento y lo plasmó así: "Yo utilizo la palabra enfermería a falta de otra mejor. Se ha limitado a significar poco más que la administración de medicamentos y la aplicación de cataplasma. Pero debería significar el uso apropiado del aire, la luz, el calor, la limpieza y su administración, y con menor gasto de energía por el paciente."’ En esos tiempos, la enfermería era una serie de tareas y técnicas subordinadas por los médicos, era un servicio humanitario compasivo y una vocación; sin embargo, la disciplina ha avanzado con pasos lentos pero firmes; ésta inició el trabajo encaminado a la producción de un cuerpo de conocimientos propio de la enfermería.

Florence entendía de higiene e hizo vínculo entre ésta y la curación. Las alcantarillas debajo del hospital fueron limpiadas y los animales muertos fueron retirados de la fuente del agua; abrió las ventanas, se lavaron pisos, paredes y la ropa de cama; los pacientes fueron aseados; así mismo se tomaron otras medidas en los alimentos; todos estos cambios debían ser aplicados a hospitales y casas para mejorar la salud de los pacientes.

Fue pionera en proponer y demostrar los efectos del ambiente en el cuidado, en la promoción de la salud de las personas, estableciéndose como marco en el inicio de la enfermería moderna. Ahora se cuenta con teorías y modelos que permiten guiar el cuidado enfermero.

La protección de los pacientes fue una preocupación constante para Nightingale; se hace evidente cuando dice: "Si un paciente tiene frío o fiebre, o está mareado o tiene una escara, la culpa, generalmente, no es de la enfermedad, sino de la enfermería."" "La verdadera enfermería ignora la infección si no es para prevenirla." "Toda enfermera debe lavarse las manos con frecuencia a lo largo del día." 2

Se puede identificar que la seguridad del paciente y la prevención de úlceras por presión son dos de sus premisas. La seguridad del paciente no figura como tal; pero en la actualidad reducir el riesgo de salud de las infecciones nosocomiales ${ }^{3}$ es una de las metas internacionales de seguridad del paciente. Por otra parte, la prevención de úlceras por presión ${ }^{4}$ es uno de los indicadores principales de la calidad de atención de la enfermería. Los profesionales de enfermería desempeñan un papel vital, ya que son los encargados de establecer un plan de cuidados a fin de prevenir la aparición de úlceras por presión y acelerar la recuperación del paciente cuando éstas ya están establecidas. Como se puede observar, estas dos premisas siguen vigentes como cuando ella las observó y documentó.
Ahora bien, la vocación fue importante para Nightingale: “... nuestra vocación de enfermera, ella es esencial, porque con seguridad puede afirmarse no que el hábito de observar correcta y prontamente nos vaya a hacer por sí mismo enfermeras útiles, sino que sin él seríamos inútiles, a pesar de nuestra entrega". ${ }^{2}$

Enfermería es una de las profesiones que cuentan con un mayor número de profesionales que acceden a estos estudios por vocación, porque sienten la necesidad de ayudar a los demás, sobre todo en los momentos más duros de la vida. ${ }^{5,6}$ Nightingale concibió algunas bases en su libro de notas; sin embargo, en la segunda mitad del siglo pasado, aproximadamente, la profesión se ha revisado, con afán de reconocer las características del surgimiento y desarrollo de la enfermería.

En este sentido M. Hall ha considerado algunas particularidades de la enfermería, ${ }^{5}$ entre las que se destacan las siguientes: constituye un servicio a la comunidad, posee un cuerpo de conocimientos propio, se encarga de preparar a las personas que van a desempeñarla, establece sus propias normas, adapta sus servicios a las necesidades que se van prestando, acepta la responsabilidad de proteger al público al que sirve, busca el bienestar y la felicidad de quienes la ejercen, está motivada más por el compromiso con la causa a la que sirve, antes que por consideraciones de tipo económico, se ajusta a un código de conducta basado en principios éticos, convoca la unión de sus miembros con el propósito de alcanzar fines comunes y se gobierna a sí misma.

Por otra parte, Nightingale subrayó que la observación y la reflexión son herramientas medulares en la enfermería: "Si no puedes conseguir el hábito de la observación de una forma u otra, mejor es que renuncies a ser enfermera, porque no es tu vocación, a pesar de lo amable que seas y lo ansiosa que puedas estar para conseguirlo." "La lección práctica más importante que puede darse a las enfermeras es enseñarles a observar -cómo observar, qué síntomas indican una mejoría del enfermo, cuáles lo contrario, cuáles tienen importancia y cuáles no, cuáles son señal evidente de negligencia y qué clase de negligencia-."2

La observación toma un significado y un valor como uno de los elementos que orientan la atención hacia el paciente; consiste en el uso de los sentidos para adquirir información sobre el paciente, sus seres queridos, el entorno y las interacciones entre éstos. Es una habilidad que exige disciplina y práctica; demanda una amplia base de conocimientos y el uso consciente de los sentidos.

Esa capacidad de observación junto con la interpretación que hacemos de la situación sirven como elementos definitivos, no sólo para brindar el cuidado, sino para evaluar el cómo y por qué se brindó esa atención de enfermería. 
Referente a la administración escribió: "Estar al frente, o ser la persona responsable, ciertamente no es sólo cumplir las propias tareas, sino procurar que todos los demás lo hagan también; (...) tampoco se trata - repito- de hacerlo todo por sí mismo, ni de distribuir los deberes a cada una de las personas a su cargo, sino de asegurarse de que cada una de ellas cumpla el deber que se le asignó."

La visión administrativa en esa época era precaria; sin embargo, ya se observaban matices. Hoy, el profesional de enfermería debe ser un líder que posea sabiduría, poder y ganas de transformar el trabajo; así mismo, capaz de compartir responsabilidades en pro de la persona enferma. Finalmente, se puede concluir que Florence Nightingale con su libro Notas de Enfermería sentó las bases de la enfermería profesional; es una obra adelantada a su época; algunos de sus conceptos, que se reinterpretaron, han crecido y se han transformado, pero continúan vigentes después de siglo y medio de haber sido escritos.

\section{BIBLIOGRAFÍA}

1. Attewe A. Florence Nightingale. Perspectivas: Revista trimestral de educación comparada. 1998; 28 (1): 173-189.

2. Nightingale F. Notas sobre Enfermería: qué es y qué no es. $2^{a}$ ed. México: Salvat Editores (original publicado en 1946); 1991.

3. Joint Commission International Accreditation Standards for Hospitals. $4^{\text {th }}$ edition. Joint Commission International; 2010. pp. 33-232.

4. Secretaría de Salud. Tres nuevos indicadores para la prevención de infecciones y seguridad del paciente. México: Secretaría de Salud; 2006. pp. 35-49.

5. Cárdenas L. La profesionalización de la enfermería en México. Un análisis desde la sociología de las profesiones. México: Ediciones Pomares; 2005. pp. 4-32, 235-260.

6. Maceira J, Martín E, Terry I. Nivel de vocación por la especialidad de enfermería en los estudiantes de segundo año. Rev Cubana Enfermer. 1999; 15 (1): 17-2. 